\title{
ON THE SOLIDIFICATION OF DENDRITIC ARRAYS: SELECTION OF THE TIP CHARACTERISTICS OF SLENDER NEEDLE CRYSTALS BY ARRAY INTERACTIONS
}

\author{
B. J. SPENCER $\dagger$ and H. E. HUPPERT \\ Institute of Theoretical Geophysics, Department of Applied Mathematics and Theoretical Physics, \\ University of Cambridge, Silver Street, Cambridge, CB3 9EW, U.K.
}

(Received 18 July 1997; accepted in revised form 21 November 1997)

\begin{abstract}
We obtain a unique solution to the well known indeterminacy for Ivantsov dendrites [Dokl. Akad. Nauk. SSSR, 58, 567 (1947)] by considering the directional solidification of a binary alloy as an array of interacting needle crystal dendrites. From the results of an asymptotic theory for the steady-state solidification of slender needle crystal arrays, the shape of the dendrite can be obtained from the solution of a non-linear integral equation. Here we solve this integral equation numerically to determine the characteristics of the solutions and compare the results to dendrite morphologies observed in experiments. The integral equation has a solvability condition that selects a distinct tip radius and tip undercooling for a given set of experimental conditions and dendrite spacings. This selection criteria is fundamentally different from traditional tip selection theories based on surface energy, and is linked to the interactions of dendrites in the array. Predictions of the tip radius are in good agreement with experimental measurements for conditions where the asymptotic theory is expected to be valid. Our results suggest that the tip radius increases with array spacing in the experimentally relevant parameter range. This relationship is consistent with the existence of a range of stable array spacings during directional solidification: the lower bound on array spacings is set by the stability criteria for array overgrowth, and an upper bound may be determined by the condition for tip splitting. In the asymptotic limit of small dendrite spacings, our integral equation interestingly has a degenerate set of solutions, indicating a transition from selection to degeneracy in the limit of small spacings. The explanation of this transition is beyond the scope of our theory and remains to be addressed. (C) 1998 Acta Metallurgica Inc.
\end{abstract}

\section{INTRODUCTION}

A long term goal in the modeling of solidification processes is the prediction of morphologies and length scales. In the simplest systems, such as the directional solidification of a binary alloy, we have a good understanding of the qualitative behavior of the morphologies and length scales, but a comprehensive quantitative theory is still being actively sought $[1,2]$.

In [3], we presented an asymptotic analysis of a model for the directional solidification of a binary alloy as an array of three-dimensional needle crystals. In this analysis, we specifically chose parameter scalings to correspond to the conditions of dendritic growth. The result of the analysis is a one-dimensional non-linear integral equation for the shape of the needle crystal. In this paper we solve this integral equation numerically to determine the steadystate shapes as a function of the solidification conditions.

Our numerical method reduces the integral equation to a non-linear system of equations for the

$†$ Present address: Department of Mathematics, SUNY at Buffalo, NY 14214-3094, U.S.A. shape at node points along the length of the dendrite. We solve the system of non-linear equations numerically using a linearized iterative scheme. A particular feature of the system of equations, which is due to the physics incorporated into the original integral equation, is that there is a solvability condition: for a given set of experimental parameters and dendrite spacings, there is a selection of the tip radius, tip undercooling and shape of the dendrite.

Our theory has no adjustable parameters. Predictions of the tip radius are in good agreement with experimental measurements over a range of solidification velocities. The selection of the tip radius in our theory is due to the interactions of the dendrites in the array and is entirely independent of surface energy. As such, it represents a fundamental philosophical alternative to tip selection theories such as marginal stability theory [4], microsolvability theory [5,6] or interfacial wave theory [7], in which surface energy is responsible for selection of an Ivantsov dendrite [8].

Another feature of our results is that the predicted tip radius increases monotonically with the dendrite spacing for the typical spacings observed in experiments. We suggest that this relationship is 
consistent with the existence of a range of stable array spacings during directional solidification. The lower bound on stable array spacings is set by the stability criteria for array overgrowth as in [9]. An upper stability bound can be determined by the condition for tip splitting [4]. By virtue of a monotonic relationship between tip radius and dendrite spacing, the maximum stable tip radius corresponds to a maximum stable array spacing.

In the limit of zero temperature gradient our theory describes the solidification of an array of solutal dendrites, or, the equivalent problem of a single solutal dendrite growing along the axis of a tube. In this case our theory predicts that for a given tube size (or dendrite spacing), there is a unique tip radius and growth velocity for a given undercooling. As the undercooling increases so does the growth velocity. However, for infinite dendrite spacing our theory recovers the well-known Ivantsov dendrite for which the tip radius is not uniquely determined. Thus, tip selection in our theory is due to the non-linear interaction of the dendrite with neighboring dendrites (or sidewalls).

We have also found that there are actually two solutions for the same solidification conditions and array spacing. The first solution corresponds well to dendritic growth, while the second solution, having a much larger tip radius, may perhaps be a vestige of the "cellular" morphology existing well into the parameter regime for dendritic solidification.

Our results are similar in spirit to earlier work on directional solidification of dendrite arrays by Hunt [10] and Kurz and Fisher [11] which relate the dendrite spacing to the tip characteristics by assuming a priori a simple shape for the dendrite. While these papers contain a similar end result, namely a relationship between the tip characteristics and the dendrite spacing, each uses an assumed dendrite shape which does not solve the free boundary problem for solidification. In our work we determine the relationship between the tip characteristics and the spacing by solving the detailed problem for the dendrite shape. If one uses a tip selection criteria based on surface energy, then the Hunt theory gives a prediction of a unique dendrite spacing [12]. The same selection criteria could also be applied to our results to obtain a unique spacing. Recent experimental evidence, however, suggests that there is not a unique dendrite spacing during directional solidification, but rather a range of stable spacings $[13,14]$ and we have interpreted our theory in light of these observations.

Our work also contains similarities to the description of steady-state dendrite arrays by Warren and Langer [9]. Both treat dendrites as line sources of solute, but the dendrite shapes assumed by Warren and Langer do not satisfy the free boundary problem. Their solutions also contain one more degree of freedom than ours. Without surface energy the Warren and Langer solutions have two degrees of freedom, and by using a selection criterion based on surface energy for the tip characteristics they obtain a family of solutions parameterized by the dendrite spacing. We also obtain a family of solutions parameterized by the spacing, but this is due to a tip selection mechanism intrinsic to the free boundary problem for the array and without recourse to a selection criterion based on surface energy. In our theory, surface energy is not responsible for selection of a unique tip radius, but rather is responsible for determining the range of tip radii which are stable from a family of permissible solutions.

The crucial role of the dendrite spacing in determining a unique solution is revealed by comparing our results for dendrite morphologies with previous work on cellular morphologies $[15,16]$. The cellular work considers directional solidification in the limit of cell spacing much less than the diffusion length and finds that in the absence of surface tension the solutions to the free boundary problem are characterized by two degrees of freedom corresponding to the spacing and the tip undercooling. Our work focuses on dendrite morphologies where the spacing and diffusion length are comparable in magnitude. In this different limit the solutions have one less degree of freedom, being characterized by a dendrite spacing with the tip undercooling determined as part of the solution. The difference in the number of degrees of freedom in the solutions for the cellular and dendrite limiting cases can be reconciled through our integral equation. We show that in the limit of small spacings the solution to our integral equation has two degrees of freedom instead of one, in agreement with the cellular results $[15,16]$. Thus, the selection of solutions in our theory is due to interaction effects present in the free boundary problem when the dendrite spacing is comparable to the diffusion length and not in the limiting case when the spacing is small compared to the diffusion length.

From the above discussion, our integral equation apparently has a transition in the number of degrees of freedom in the limit of small spacings. Numerically we find that for fixed, order unity spacing there is a selected tip state. If we consider our finite-spacing model and evaluate the asymptotic limit of small spacings, the simplified integral equation has a degenerate set of solutions corresponding to computed cellular morphologies $[15,16]$. Unfortunately, our numerical method fails in the limit of small spacings, so we are not able to describe this apparent transition in the number of degrees of freedom as the spacing approaches zero. We have also not yet been able to find a satisfying physical mechanism for why such a transition might occur. It has been suggested by a referee that in the absence of a description of this transition, our model lacks a crucial link which validates the presence of a selection mechanism at finite spacings, 
and consequently our calculations may be incorrect. We are of the opinion that such a transition is a common occurrence in non-linear systems, and that the validity of our model and calculations can be easily confirmed.

A related set of calculations on dendrite arrays has been performed by $\mathrm{Lu}$ and Hunt [17]. Our work should be viewed as complementary to their model in the sense that our work is relevant to the description of slender morphologies at moderate to large dendrite spacings which their calculations would have difficulty describing. Their calculations also give a unique shape for a given dendrite spacing. However, this result appears to depend on the presence of surface energy. In particular, the existence of a dendritic solution in their model depends on having an anisotropic surface energy. Aside from a fundamental difference regarding the role of surface energy, their calculations contain similar features to our work, namely, a range of stable array spacings which can be bounded by overgrowth and tip splitting conditions, as well as the appearance of two distinct types of solutions.

The rest of the paper is organized as follows. In Section 2 we present a brief summary of the integral equation developed in [3]. In Section 3 we describe the numerical method and solvability condition used to obtain solutions to the integral equation. In Section 4 we compare our theory to experimental observations of dendritic growth and indicate its relevance to the determination of the range of stable spacings during dendritic growth. Our main conclusions are summarized in Section 5.

\section{AN INTEGRAL EQUATION FOR THE DIRECTIONAL SOLIDIFICATION OF SLENDER NEEDLE CRYSTAL ARRAYS}

We consider the directional solidification of a binary alloy of composition $C_{\infty}$ with a phase diagram as given in Fig. 1. The alloy has initial freezing temperature $T_{0}$ and equilibrium freezing range $\Delta T_{0}$. For the purposes of simplicity, the phase diagram is assumed to consist of straight lines with constants $k$ and $m_{\mathrm{L}}$ denoting the segregation coefficient and liquidus slope, respectively. The freezing range for the alloy is then given by $\Delta T_{0}=m_{\mathrm{L}} C_{\infty}(k-1) / k$.

Figure 2 illustrates the directional solidification of an array of smooth needle crystals. The solidification front moves in the $-z$ direction at constant speed $V$. The system is modeled using the one-sided model for directional solidification [18] in which diffusion of solute in the solid is neglected and the temperature field is given by the "frozen temperature" approximation. The full details of the model are given in [3]. The "frozen temperature" approximation assumes that the diffusion of heat is much faster than the diffusion of solute in the liquid, and that the diffusivity of heat in the liquid and the

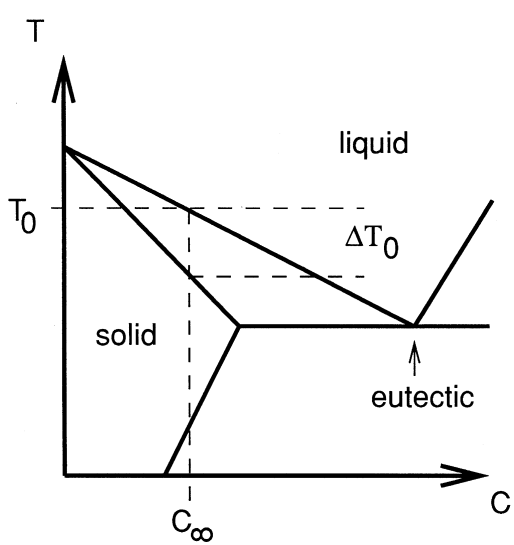

Fig. 1. Phase diagram for a binary alloy.

solid is the same. In a coordinate frame moving with the solidification front, the imposed linear temperature profile is then given by

$$
T=T_{0}-G z,
$$

where $T_{0}$ is the reference temperature defining $z=0$ (the liquidus isotherm for composition $C_{\infty}$ ), and $G$ is a constant, positive temperature gradient (it is warmer ahead of the front). We assume the solidification morphology consists of a periodic array of identical, smooth needle crystals with rectangular planform as depicted in Fig. 2. There are four length scales used to quantify the morphology. The first is the position of the tips relative to the reference temperature isotherm, $z_{\text {tip }}$, which is directly related to the tip undercooling by the temperature gradient. The second is the tip radius $\rho$ given by the harmonic mean of the two principle radii of curvature of the dendrite tip. Finally, the dendrite spacings $\lambda_{x}$ and $\lambda_{y}$ are the spacings of the

(a) side view

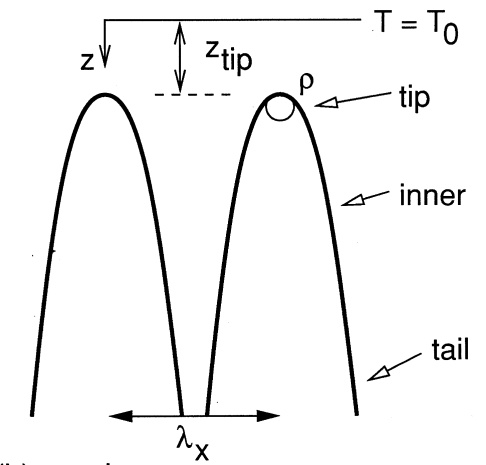

(b) top view

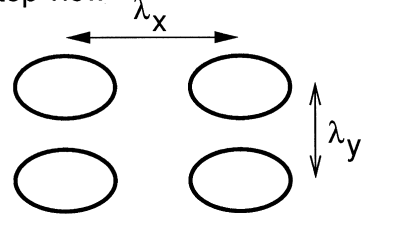

Fig. 2. Schematic of array of needle crystals: (a) side view; (b) top view. 
rectangular array in the $x$ and $y$ directions, respectively.

In addition to these four morphological length scales, there are three fundamental process length scales which are determined by the solidification conditions [2]. The first length scale is the diffusion length $l_{\mathrm{D}}=D / V$ where $D$ is the diffusivity of solute in the liquid and $V$ is the imposed solidification speed. The second length scale is a thermal length, $l_{\mathrm{T}}=\left(k \Delta T_{0}\right) / G$, which is related to the vertical extent of equilibrium freezing in the imposed temperature gradient. The last process length scale is the capillary length $l_{\mathrm{C}}=\Gamma /\left(k \Delta T_{0}\right)$. The capillary constant $\Gamma$ relates the melting temperature of a curved solid/ liquid interface $T_{\kappa}$ to that of a flat interface $T_{\mathrm{f}}$ according to the Gibbs-Thomson equation $T_{\kappa}=T_{f}-\Gamma^{\kappa}$ where $\kappa$ is the curvature of the interface.

In [3] the relative sizes of the different length scales for dendritic solidification are determined from a study of experimental data. This analysis suggests asymptotic scalings in terms of a small non-dimensional parameter

$$
\epsilon=l_{\mathrm{D}} / l_{\mathrm{T}} \ll 1
$$

For a given set of solidification conditions $\epsilon$ is known (a control parameter). The scalings for the different length scales are

$$
\begin{gathered}
\rho / l_{\mathrm{D}}=\epsilon P, \\
\left(\lambda_{x}, \lambda_{y}\right) / l_{\mathrm{D}}=\left(\Lambda_{x}, \Lambda_{y}\right),
\end{gathered}
$$

and

$$
l_{\mathrm{C}} / l_{\mathrm{D}} \ll \epsilon^{2},
$$

where $P, \Lambda_{x}$, and $\Lambda_{y}$ are taken to be $O(1)$. In the above equations, $P$ corresponds to a scaled tip radius parameter, and $\Lambda_{x}, \Lambda_{y}$ are non-dimensional dendrite spacings. Scaling (5) leads to a dendrite shape which is independent of surface energy to first approximation. The scaled tip position $\mathcal{Z}$ is given by

$$
\mathcal{Z}=\delta z_{\text {tip }} / l_{\mathrm{D}},
$$

where $\delta$ is a parameter related directly to $\epsilon$ by

$$
\delta=\frac{1}{\ln (1 / \epsilon)} .
$$

In the asymptotic theory we view $\delta$ as an order unity constant even for the smallest realistic values of $\epsilon$ because of the logarithmic dependence.

The dendrite shape is described in cylindrical coordinates $(r, z, \theta)$ relative to the center axis of the dendrite. We describe the dendrite shape with nondimensional variables using the diffusion length $l_{\mathrm{D}}$ as our length scale. The surface of the dendrite is then given by

$$
r=l_{\mathrm{D}} R(\zeta, \theta),
$$

where $\zeta$ is the non-dimensional distance behind the dendrite tip given by

$$
\zeta=\frac{z-z_{\text {tip }}}{l_{\mathrm{D}}}
$$

In [3], we present a detailed asymptotic analysis of the free boundary problem for the dendrite shape. We solve the problem to determine the first order solution (neglecting corrections of order $\epsilon$ ) using slender body theory and matched asymptotic expansions [19]. The shape of the dendrite is described in three regions: a tip region $[\zeta=O(\epsilon) \ll 1]$, an inner region $[\zeta=O(1)]$, and a tail region $[\zeta=O(1 / \epsilon) \gg 1]$. The three locally-valid solutions are matched to each other to generate a uniformly valid composite solution for the whole dendrite:

$$
R=R_{\text {tip }}+R_{\text {in }}-R_{\text {tip } / \text { in }}+R_{\text {tail }}-R_{\text {tail } / \text { in }} .
$$

In the above, the tip solution, $R_{\text {tip }}$, and the solution common to the matched tip and inner solutions, $R_{\text {tip/in }}$, are identically given by an Ivantsov dendrite with a scaled tip radius $P$,

$$
R_{\mathrm{tip}}=R_{\mathrm{tip} / \mathrm{in}}=\sqrt{2 \epsilon P \zeta} .
$$

The solution common to the matched tail and inner solutions is given by

$$
R_{\mathrm{tail} / \mathrm{in}}=\sqrt{\epsilon \frac{\Lambda_{x} \Lambda_{y}}{\pi} \zeta} .
$$

The solution in the tail region, $R_{\text {tail }}$, is not axisymmetric and not known explicitly. However, the cross-sectional area of the tail solution is known from conservation of solute to be

$$
A_{\text {tail }}=\Lambda_{x} \Lambda_{y}\left\{1-[1+(1-k) \epsilon \zeta]^{-1 /(1-k)}\right\} .
$$

In addition, the liquid domain is truncated by the formation of the eutectic phase at

$$
\zeta_{\mathrm{E}}=\frac{1}{\epsilon} \frac{C_{\mathrm{E}}-C_{\infty}}{C_{\infty}(1-k)},
$$

where $C_{\mathrm{E}}$ is the eutectic composition. By equation (12), the only information passed from the tail region to the rest of the solution is the area each dendrite occupies in the periodic array, $A_{\Lambda}=\Lambda_{x} \Lambda_{y}$. Thus, the details of the tail shape are not necessary for determining the behavior of the dendrite tip. The details of the tail region merely describe how the rectangular planform is filled as solidification proceeds along the length of the dendrite subject to the constraints (13) and (14). Because these tail details do not affect the rest of our solution, for the purposes of illustrating the entire composite solution we shall approximate $R_{\text {tail }}$ by the effective radius $R_{\text {tail }}^{\text {eff }}$ of the cross-sectional area of the tail from (13) as

$$
R_{\text {tail }} \approx R_{\text {tail }}^{\text {eff }}=\sqrt{A_{\text {tail }} / \pi}
$$

This effective radius corresponds to the axisym- 
metric tail shape of a dendrite solidified in a cylindrical tube of area $A_{\Lambda}$ with radius

$$
R_{\Lambda}=\sqrt{\Lambda_{x} \Lambda_{y} / \pi} .
$$

The true composite solution should have an $R_{\text {tail }}$ which is not axisymmetric. However, these details would only affect our plots of the composite solution and are irrelevant to the inner and tip solutions. Our predictions of the tip radius and tip undercooling are similarly unaffected.

The determination of the inner solution, $R_{\text {in }}$, appearing in the composite solution (10) is somewhat complicated and is at the heart of the asymptotic analysis. In the inner region, the dendrite appears as a slender body with

$$
R_{\text {in }}(\zeta, \theta)=\epsilon^{1 / 2} \bar{R}(\zeta)
$$

where $\bar{R}(\zeta)$ is $O(1)$. Viewed from an $O(1)$ distance away, this slender dendrite appears to be a line source of solute of strength $q(\zeta)$ extending from $0<\zeta<\infty$. From conservation of solute at the surface of the slender dendrite one obtains the following relationship between the solute source strength and the dendrite shape,

$$
\bar{R}(\zeta)=\sqrt{\frac{1}{\pi \delta} \int_{0}^{\zeta} q\left(\zeta^{\prime}\right) \mathrm{d} \zeta^{\prime}} .
$$

The line source $q(\zeta)$ satisfies the following non-linear integral equation derived from the condition of local equilibrium on the surface of the needle crystal,

$$
\begin{aligned}
\mathcal{Z} & +\delta \zeta=\frac{q(\zeta)}{4 \pi \delta}-\frac{q(\zeta)}{4 \pi}\left[\ln \left(\frac{\bar{R}^{2}}{4 \zeta}\right)+\gamma_{\mathrm{E}}\right] \\
& +\int_{0}^{\infty}\left[q\left(\zeta^{\prime}\right)-q(\zeta)\right] G_{\text {near }}\left(\zeta ; \zeta^{\prime}\right) \mathrm{d} \zeta^{\prime} \\
& +\int_{0}^{\infty} q\left(\zeta^{\prime}\right) G_{\text {array }}\left(\zeta ; \zeta^{\prime}\right) \mathrm{d} \zeta^{\prime},
\end{aligned}
$$

where $\gamma_{\mathrm{E}}$ is Euler's constant,

$$
G_{\text {near }}\left(\zeta ; \zeta^{\prime}\right)=\frac{1}{4 \pi} \frac{\exp \left\{-(1 / 2)\left[\left|\zeta^{\prime}-\zeta\right|+\left(\zeta^{\prime}-\zeta\right)\right]\right\}}{\left|\zeta^{\prime}-\zeta\right|},
$$

and

$$
\begin{aligned}
& G_{\text {array }}\left(\zeta ; \zeta^{\prime}\right)=\sum_{i^{2}+j^{2} \neq 0} \sum \frac{1}{4 \pi} \\
& \frac{\exp \left\{-(1 / 2)\left[\sqrt{\left(i \Lambda_{x}\right)^{2}+\left(j \Lambda_{y}\right)^{2}+\left(\zeta^{\prime}-\zeta\right)^{2}}+\left(\zeta^{\prime}-\zeta\right)\right]\right\}}{\sqrt{\left(i \Lambda_{x}\right)^{2}+\left(j \Lambda_{y}\right)^{2}+\left(\zeta^{\prime}-\zeta\right)^{2}}} .
\end{aligned}
$$

In the above equations, $G_{\text {near }}$ and $G_{\text {array }}$ follow from the Green's function for solute diffusion in a moving frame of reference. The last integral in equation (19) is the contribution to the solute field near the "center" dendrite that is generated by all the other dendrites in the array. Solutions to the integral equation must satisfy matching conditions with the tip and tail solutions. Matching in the tail region requires

$$
q(\infty)=\delta \Lambda_{x} \Lambda_{y},
$$

which is an imposed constraint if we prescribe the spacings. Solutions to the integral equation automatically satisfy this condition because as $\zeta \rightarrow \infty$ the dominant terms of (19) are

$$
\delta \zeta \sim \int_{0}^{\infty} q\left(\zeta^{\prime}\right) G_{\text {array }}\left(\zeta ; \zeta^{\prime}\right) \mathrm{d} \zeta^{\prime} \text { as } \zeta \rightarrow \infty
$$

which is only satisfied if $q(\zeta) \backsim \delta \Lambda_{x} \Lambda_{y}$ as $\zeta \rightarrow \infty$. Matching the inner solution to the tip solution requires

$$
P=\frac{q(0)}{2 \pi \delta} .
$$

Equation (24) thus links the dendrite tip radius to the strength of the solute source at the tip.

The preceding description of the solution has complicated details, but the idea is straightforward. The determination of the composite solution (10) hinges on finding $R_{\text {in }}$. To determine $R_{\text {in }}$ we must solve the integral equation (19) for $q(\zeta)$. The parameters appearing in this equation are $\delta, \Lambda_{x}$ and $\Lambda_{y}$. For a given set of experimental conditions $\left(C_{\infty}\right.$, $V, G$, and material parameters) the parameter $\delta$ is prescribed. Array spacings $\lambda_{x}$ and $\lambda_{y}$ appear as the spacing parameters $\Lambda_{x}$ and $\Lambda_{y}$ in $G_{\text {array. Arrays of }}$ different spacings are described by varying $\Lambda_{x}$ and $\Lambda_{y}$. The unknowns we need to determine are the source strength $q(\zeta)$, the scaled tip radius $P$, and the scaled tip position $\mathcal{Z}$. By substituting for the $\bar{R}$ term in the integral equation (19) and using equation (18), we have a single equation for $q(\zeta)$ and $\mathcal{Z}$ in terms of the parameters $\delta, \Lambda_{x}$ and $\Lambda_{y}$. We solve the non-linear integral equation to find $q(\zeta)$ and $\mathcal{Z}$ simultaneously. The scaled tip radius for the dendrite is then given by (24). From $q(\zeta)$ we find the inner solution $R_{\text {in }}$ from (18) and (17). Knowing $R_{\text {in }}$ and $P$, we can then calculate a composite solution for the dendrite shape from equations (10)-(15). The dimensional tip radius and tip undercooling of our solution is thus given directly by

$$
\rho=\epsilon P l_{\mathrm{D}}
$$

and

$$
\Delta T=T_{0}-T_{\text {tip }}=G \mathcal{Z} l_{\mathrm{D}} / \delta .
$$

A simplified explanation as to why the spacing of the array selects a tip radius is as follows [3]. Consider the directional solidification of a single dendrite along the center of a cylindrical tube of dimensional radius $R_{\lambda}$ in a weak temperature gradient for which $l_{\mathrm{D}} \ll l_{\mathrm{T}}$. Here, $2 R_{\lambda}$ mimics the dendrite 
spacing $\lambda$. Provided the walls are far enough away, we can describe the dendrite tip in dimensional variables as an Ivantsov paraboloid

$$
R_{\text {tip }}^{2}=2 \rho\left(z-z_{\text {tip }}\right)
$$

with

$$
\frac{T_{0}-T_{\text {tip }}}{k \Delta T_{0}}=\left(\rho / 2 l_{\mathrm{D}}\right) E_{1}\left(\rho / 2 l_{\mathrm{D}}\right) \exp \left(\rho / 2 l_{\mathrm{D}}\right) .
$$

In the region far behind the tip, $\left(z-z_{\text {tip }}\right)=O\left(l_{\mathrm{T}}\right)$ and the shape is controlled by the weak temperature gradient and the nearness of the dendrite surface to the tube walls. The slowly varying Scheiltype solution for the tail region can be determined from conservation of mass to be

$$
R_{\text {tail }}^{2}=R_{\lambda}^{2}\left\{1-\left[1+(1-k)\left(z-z_{\text {tip }}\right) / l_{\mathrm{T}}\right]^{-1 /(1-k)}\right\} .
$$

The basic idea behind the selection mechanism is that there is only one self-consistent solution for the shape which describes the transition from the Ivantsov tip to the Scheil-type tail. In our full model this transition is accomplished by the inner solution and requires the solution of the integral equation (19). For this simplified explanation the analogy is requiring the Ivantsov tip (27) and Scheil-like tail (29) to match in a region in between the tip and tail regions, where $\rho \ll z-z_{\text {tip }} \ll l_{\mathrm{T}}$. In this region the tail solution behaves like

$$
R_{\text {tail }}^{2} \sim R_{\lambda}^{2} \frac{z-z_{\text {tip }}}{l_{\mathrm{T}}} .
$$

Hence, matching to the Ivantsov tip requires

$$
\frac{R_{\lambda}^{2}}{l_{\mathrm{T}}}=2 \rho .
$$

Thus, since we know $l_{\mathrm{T}}$ from the temperature gradient, (31) gives a unique $\rho$ for each "spacing" $R_{\lambda}$. While the above illustrates the idea of what is underlying tip selection in our theory, the relationship (31) is not correct because the two solution pieces in our simple model, even when matched, do not solve the free boundary problem. To determine the correct relationship between the tip radius and the dendrite spacing, one must correctly solve for the transitional inner shape $\bar{R}$ which connects the tip and tail solutions. This solution for $\bar{R}$ necessarily involves the interaction of the shape with the neighboring dendrites in the array, and thus is somewhat complicated, but the underlying mechanism for selection is the same.

\section{NUMERICAL SOLUTIONS TO THE INTEGRAL EQUATION}

To determine the dendrite shape we solve the integral equation (19) for $q(\zeta)$ by discretizing the domain $0<\zeta<\infty$ into $N$ subintervals and solving for the unknown source strength $q(\zeta)$ at the $N+1$ node points. The resulting non-linear system of equations has a solvability condition which determines $\mathcal{Z}$. To solve the non-linear system we employ an iterative technique based on linearizing the problem about a trial solution, coupled with a singular value decomposition of the resulting linear problem to facilitate the implementation of the solvability condition. Readers not interested in the details of the numerical method could skip forward to Section 4.

We first map the infinite domain $0<\zeta<\infty$ to $0<s<1$ using the transformation

$$
s=\frac{1}{1+\zeta / \alpha},
$$

where $\alpha$ is a (constant) stretching parameter which can be chosen to accommodate the behavior of the solution for large $\zeta$. The mapping takes the tip $(\zeta=0)$ to $s=1, \zeta=\alpha$ to $s=1 / 2$, and the tail $(\zeta=\infty)$ to $s=0$. In principle, the precise value of $\alpha$ should not affect the dendrite shape. However, for solutions based on a finite number of points $N$, extreme values of $\alpha$ cause the numerical method to fail and/or converge poorly. If $\alpha$ is too large the dendrite tip is poorly resolved. If $\alpha$ is too small the behavior in the tail region is poorly resolved and the solvability method fails. For small $N$, discrete solutions depend weakly on $\alpha$, but for finite $\alpha$ and sufficiently large $N$ the results are independent of the choice of $\alpha$. We take $\alpha=5$ for all our calculations as a compromise which balances the resolution of the tip and tail regions, and take $N$ sufficiently large to obtain results that are independent of $\alpha$.

Corresponding to the mapped domain $0<s<1$, we define the mapped functions

$$
\begin{gathered}
Q(s)=q(\zeta) / \delta, \\
f(s)=\frac{(\bar{R}(\zeta))^{2}}{4 \zeta}, \\
g_{\mathrm{N}}\left(s ; s^{\prime}\right)=G_{\text {near }}\left(\zeta ; \zeta^{\prime}\right),
\end{gathered}
$$

and

$$
g_{\mathrm{A}}\left(s ; s^{\prime}\right)=G_{\text {array }}\left(\zeta ; \zeta^{\prime}\right) .
$$

The mapped integral equation becomes

$$
\begin{aligned}
\mathcal{Z}+\delta \alpha(1 / s-1)= & \frac{Q(s)}{4 \pi}-\delta \frac{Q(s)}{4 \pi}\left[\ln f(s)+\gamma_{\mathrm{E}}\right] \\
& +\delta I_{\mathrm{N}}(s)+\delta I_{\mathrm{A}}(s)
\end{aligned}
$$

where

$$
\begin{gathered}
f(s)=\frac{s}{4 \pi(1-s)} \int_{s}^{1} \frac{Q\left(s^{\prime}\right)}{\left(s^{\prime}\right)^{2}} \mathrm{~d} s^{\prime}, \\
I_{\mathrm{N}}(s)=\int_{0}^{1}\left[Q\left(s^{\prime}\right)-Q(s)\right] \frac{\alpha g_{\mathrm{N}}\left(s ; s^{\prime}\right)}{\left(s^{\prime}\right)^{2}} \mathrm{~d} s^{\prime},
\end{gathered}
$$

and 


$$
I_{\mathrm{A}}(s)=\int_{0}^{1} Q\left(s^{\prime}\right) \frac{\alpha g_{\mathrm{A}}\left(s ; s^{\prime}\right)}{\left(s^{\prime}\right)^{2}} \mathrm{~d} s^{\prime} .
$$

The mapped integral equation (37) is singular at $s=0$. However, we know from the tail condition (22) that $Q(0)=\Lambda_{x} \Lambda_{y}$.

We discretize the mapped domain $0<s<1$ using $N$ equal subintervals. The subintervals are separated by $N+1$ node points given by

$$
s_{j}=j / N \text { for } j=0,1, \ldots, N .
$$

We denote the value of a function at the node point $j$ with a subscript, so that $Q_{j}=Q\left(s_{j}\right), f_{j}=f\left(s_{j}\right)$, $\left(I_{N}\right)_{j}=I_{N}\left(s_{j}\right)$, and $\left(I_{\mathrm{A}}\right)_{j}=I_{\mathrm{A}}\left(s_{j}\right)$. The tail condition (22) gives

$$
Q_{0}=\Lambda_{x} \Lambda_{y}
$$

and the mapped integral equation (37) is evaluated at all nodes except $j=0$ to obtain

$$
\begin{aligned}
\mathcal{Z}+\delta \alpha\left(1 / s_{j}-1\right)= & \frac{Q_{j}}{4 \pi}-\delta \frac{Q_{j}}{4 \pi}\left[\ln f_{j}+\gamma_{\mathrm{E}}\right] \\
& +\delta\left(I_{N}\right)_{j}+\delta\left(I_{A}\right)_{j} .
\end{aligned}
$$

Equations (43) is expressed as a system of equations for the unknowns $Q_{j}$ by writing each integral in $f_{j}$, $\left(I_{N}\right)_{j}$ and $\left(I_{A}\right)_{j}$ [see equations (38)-(40)] in terms of the node values $Q_{j}$. We first note that the integration kernels are not well behaved in general: $f(s)$ has a singular kernel at $s^{\prime}=0, g_{\mathrm{N}}\left(s ; s^{\prime}\right)$ is singular at $s^{\prime}=s$, and $g_{A}\left(s ; s^{\prime}\right)$ changes character rapidly across $s^{\prime}=s$. However, if we assume that $Q(s)$ is relatively smooth over the interval $0<s<1$, then we can expand $Q(s)$ as a Taylor series near each node point. To illustrate, consider the generic integral

$$
I=\int_{s_{0}}^{s_{N}} Q\left(s^{\prime}\right) K\left(s^{\prime}\right) \mathrm{d} s^{\prime}
$$

where $K\left(s^{\prime}\right)$ is some known kernel. The range of integration is broken into subintervals of width $\Delta s=1 / N$ with each subinterval centered on a node point:

$$
\begin{aligned}
I= & \int_{s_{0}}^{s_{1 / 2}} Q\left(s^{\prime}\right) K\left(s^{\prime}\right) \mathrm{d} s^{\prime}+\sum_{k=1}^{N-1} \int_{s_{k-1 / 2}}^{s_{k+1 / 2}} Q\left(s^{\prime}\right) K\left(s^{\prime}\right) \mathrm{d} s^{\prime} \\
& +\int_{s_{N-1 / 2}}^{s_{N}} Q\left(s^{\prime}\right) K\left(s^{\prime}\right) \mathrm{d} s^{\prime} .
\end{aligned}
$$

In each subinterval $s_{k-1 / 2}<s^{\prime}<s_{k}+1 / 2$ we expand $Q\left(s^{\prime}\right)$ using a Taylor series

$$
Q\left(s^{\prime}\right)=Q\left(s_{k}\right)+\left(s^{\prime}-s_{k}\right) Q^{\prime}\left(s_{k}\right)+\ldots,
$$

then substitute the expansion into each integral of (45) and retain the leading order term. Thus, the integral $I$ is approximated by

$$
\begin{aligned}
I \approx & Q_{0} \int_{s_{0}}^{s_{1 / 2}} K\left(s^{\prime}\right) \mathrm{d} s^{\prime}+\sum_{k=1}^{N-1} Q_{k} \int_{s_{k-1 / 2}}^{s_{k+1 / 2}} K\left(s^{\prime}\right) \mathrm{d} s^{\prime} \\
& +Q_{N} \int_{s_{N-1 / 2}}^{s_{N}} K\left(s^{\prime}\right) \mathrm{d} s^{\prime} .
\end{aligned}
$$

While the kernels $K\left(s^{\prime}\right)$ may not be well behaved in our problem, the kernels are known explicitly. Furthermore, the subinterval integrations indicated on the right side of equation (47) can all be evaluated explicitly for each of the kernels in $f(s), I_{N}(s)$ and $I_{A}(s)$. Using this technique, we can write the integrals $f_{j},\left(I_{N}\right)_{j}$ and $\left(I_{A}\right)_{j}$ as weighted linear sums of the unknowns $Q_{k}$ as

$$
\begin{gathered}
f_{j} \approx \sum_{k=0}^{N} \mathcal{F}_{j k} Q_{k}, \\
\left(I_{N}\right)_{j} \approx \sum_{k=0}^{N} \mathcal{N}_{j k} Q_{k},
\end{gathered}
$$

and

$$
\left(I_{A}\right)_{j} \approx \sum_{k=0}^{N} \mathcal{A}_{j k} Q_{k},
$$

where $\mathcal{F}_{j k}, \mathcal{N}_{j k}$ and $\mathcal{A}_{j k}$ are coefficient matrices determined by integrating the kernels over the $k$ th subinterval. Substituting the approximations (48)(50) into equation (43) and combining with equation (42), we obtain a non-linear system of $N+1$ equations for the $N+1$ unknowns $Q_{j}$. In addition, $\mathcal{Z}$ is also unknown. As will be shown in equation (67), $\mathcal{Z}$ is determined by a solvability condition.

Since the non-linearity is due to the logarithm term of (43), the non-linearity is weak. We can thus formulate the non-linear system of equations for $Q$ as a linear system in which the matrix coefficients depend weakly on the unknown solution $\mathbf{Q}=\left(Q_{0}\right.$, $Q_{1}, \ldots, Q_{N}$ ). This formulation is represented in compact form by

$$
\sum_{k=0}^{N} A_{j k}(\mathbf{Q}) Q_{k}=\bar{b}_{j}+\mathcal{Z} b_{j}^{*} \text { for } j=0,1, \ldots, N,
$$

where $A_{j k}(\mathbf{Q})$ is the matrix of weakly varying coefficients, $\bar{b}_{j}$ and $b_{j}^{*}$ are known constants, and the $j=0$ equation corresponds to (42). Finding a discrete solution to the integral equation is equivalent to solving the non-linear system (51).

Say for the moment we ignore the weak dependence of $A_{j k}$ on $\mathbf{Q}$ and determine the matrix coefficients using $A_{j k}\left(\mathbf{Q}^{0}\right)$ where $\mathbf{Q}^{0}$ is a reasonable approximation to our desired solution $\mathbf{Q}$. This leads to an inhomogeneous linear system for $\mathbf{Q}$,

$$
\sum_{k=0}^{N} A_{j k}\left(\mathbf{Q}^{0}\right) Q_{k}=\bar{b}_{j}+\mathcal{Z} b_{j}^{*} \text { for } j=0,1, \ldots, N .
$$


The Fredholm alternative theorem states that (52) has a solution only if the homogeneous problem,

$$
\sum_{k=0}^{N} A_{j k}\left(\mathbf{Q}^{0}\right) Q_{k}=0 \text { for } j=0,1, \ldots, N,
$$

has only the trivial solution $\mathbf{Q}=0$. In this case the solution to (52) is unique and exists for any righthand side. Conversely, if the homogenous problem (53) has a non-trivial solution, then solutions to the inhomogeneous problem (52) exist only if the righthand side satisfies a solvability condition, and the resulting solution is not unique.

In the context of our solutions for the dendrite shape, if the homogeneous problem (53) has only the trivial solution then solutions exist for any $\mathcal{Z}$. We could vary $\mathcal{Z}$ to generate a family of solutions $Q(s)$ with different tip radii determined by $P=q(0) /(2 \pi \delta)=Q(1) /(2 \pi)$. Presumably we could choose a $\mathcal{Z}$ that would give us a tip radius consistent with an externally imposed tip selection criteria (e.g. marginal stability). This is the philosophy used in [9] but with a family of dendrite shapes constrained to be paraboloids. If, however, (53) has a non-trivial solution, then for solutions to (52) to exist we must choose $\mathcal{Z}$ to satisfy the solvability condition. The solution $\mathbf{Q}$ to (52) is then not unique and can be adjusted by arbitrary additions of the solution to the homogeneous problem. Thus there is a fundamental issue regarding the uniqueness of $\mathcal{Z}$ and associated solutions $Q(s)$ which hinges on the existence of non-trivial solutions to the homogeneous problem. We find that for any reasonable choice of $\mathbf{Q}^{0}$ the homogeneous linear problem (53) has a non-trivial solution. Thus $\mathcal{Z}$ is necessarily determined by a solvability condition for solutions to exist.

Of course, the full problem (51) is non-linear, so the solvability theory described above is modified somewhat. If the problem was linear, as in (52), then there would be infinitely many solutions by the Fredholm solvability theory. But in the non-linear problem (51), the degree of freedom is eliminated because the matrix coefficients actually depend on the value of $\mathbf{Q}$. Varying $\mathbf{Q}$ changes the linear part of the problem, and hence the solution. It is only a special $\mathbf{Q}$ that gives the matrix coefficients consistent with $\mathbf{Q}$ being a solution to the matrix problem. Thus solutions to the non-linear system (51) will be distinct and not form a continuous family of solutions as would be the case in the linear problem. Solutions to the non-linear system (51) are therefore characterized by distinct $\mathbf{Q}$ with an associated value of $\mathcal{Z}$ determined by a solvability condition. It follows that there is a selected tip radius, tip undercooling and dendrite shape for a given set of solidification conditions and dendrite spacings.

In view of the above, we determine the solution $\mathbf{Q}$ to the non-linear problem (51) as an iterative refinement of an initial guess $\mathbf{Q}^{0}$. At each step of the iteration we solve a linear problem to find an improved solution. Let

$$
F_{j}(\mathbf{Q})=\sum_{k=0}^{N} A_{j k}(\mathbf{Q}) Q_{k}-\bar{b}_{j}-\mathcal{Z} b_{j}^{*} .
$$

Solutions to the non-linear problem are given by

$$
F_{j}(\mathbf{Q})=0 \text { for } j=0,1, \ldots, N \text {. }
$$

To derive a basis for an iterative scheme we linearize $F_{j}(\mathbf{Q})$ about some trial solution $\mathbf{Q}=\mathbf{Q}^{0}$. Thus

$$
F_{j}(\mathbf{Q}) \approx F_{j}\left(\mathbf{Q}^{0}\right)+\sum_{k=0}^{N} B_{j k}\left(\mathbf{Q}^{0}\right)\left(Q_{k}-Q_{k}^{0}\right),
$$

where

$$
B_{j k}\left(\mathbf{Q}^{0}\right)=\left.\frac{\partial F_{j}}{\partial Q_{k}}\right|_{\mathbf{Q}=\mathbf{Q}^{0}}=A_{j k}\left(\mathbf{Q}^{0}\right)+\left.\sum_{i=0}^{N} \frac{\partial A_{j i}}{\partial Q_{k}}\right|_{\mathbf{Q}=\mathbf{Q}^{0}} Q_{i}^{0} .
$$

We then use a variant of Newton's method to solve the non-linear problem by replacing (55) by the linearization (56) and rearranging to obtain a linear system for the unknowns $Q_{k}$ in which the matrix coefficients and right-hand side depend on the trial solution $\mathbf{Q}^{0}$,

$$
\sum_{k=0}^{N} B_{j k}\left(\mathbf{Q}^{0}\right) Q_{k}=\tilde{b}_{j}\left(\mathbf{Q}^{0}\right)+\mathcal{Z} b_{j}^{*}
$$

where

$$
\tilde{b}_{j}\left(\mathbf{Q}^{0}\right)=\bar{b}_{j}+\left.\sum_{i=0}^{N} \sum_{k=0}^{N} \frac{\partial A_{j i}}{\partial Q_{k}}\right|_{\mathbf{Q}=\mathbf{Q}^{0}} Q_{k}^{0} Q_{i}^{0} .
$$

Equation (58) forms the basis for a convergent iterative scheme to compute the solution to the non-linear problem. We choose an initial guess $\mathbf{Q}^{0}$, solve for the improved solution $\mathbf{Q}$, then use the new solution as an initial guess for the next iteration. The iteration proceeds until $\mathbf{Q}$ converges, at which point we have a solution to the non-linear problem.

We now turn to the solution of the linear problem (58). As mentioned previously, there is a fundamental issue as to whether there are non-trivial solutions to the corresponding homogeneous problem. We have found that non-trivial solutions do exist, which means that (i) there is a solvability condition for the right-hand side of (58) which determines $\mathcal{Z}$, and (ii) the linear problem is inherently ill-conditioned. To deal with these aspects of the problem, we solve the linear system using a singular value decomposition [20]. The singular value decomposition of matrix $\mathbf{B}$ is given by

$$
\mathbf{B}=\sum_{i=0}^{N} \sigma_{i} \mathbf{u}_{i} \mathbf{v}_{i}^{\mathrm{T}},
$$

where superscript ${ }^{\mathrm{T}}$ denotes the vector transpose, and where the singular values $\sigma_{i}$ and orthonormal singular vectors $\mathbf{u}_{i}$ and $\mathbf{v}_{i}$ satisfy $\mathbf{B v}_{i}=\sigma_{i} \mathbf{u}_{i}$. The 
singular value decomposition of matrix $\mathbf{B}$ is found using standard LAPACK numerical subroutines. Once $\sigma_{i}, \mathbf{u}_{i}$ and $\mathbf{v}_{i}$ are known, the solution to the original matrix problem from (58),

$$
\mathbf{B Q}=\tilde{\mathbf{b}}+\mathcal{Z} \mathbf{b}^{*},
$$

is given by

$$
\mathbf{Q}=\sum_{i=0}^{N} c_{i} \mathbf{v}_{i}
$$

where

$$
c_{i}=\frac{\mathbf{u}_{i}^{\mathrm{T}}\left(\tilde{\mathbf{b}}+\mathcal{Z} \mathbf{b}^{*}\right)}{\sigma_{i}} .
$$

Singular values $\sigma_{i}$ near zero indicate ill-conditioning and the possible existence of non-trivial solutions to the homogeneous problem. For reasonable choices of the system parameters the singular value spectrum consists of a smoothly decaying portion, $\sigma_{0}>\sigma_{1}>\ldots>\sigma_{N-1}>0$, and an isolated singular value $\sigma_{N} \approx 0$. This last singular value goes to zero rapidly as $N$ increases. We interpret this near-zero singular value as indicating the existence of a nontrivial solution $\mathbf{v}_{N}$ to the homogeneous problem $\mathbf{B v}_{N}=0$.

If the homogeneous problem were exactly singular, $\sigma_{N}$ would be identically zero and the corresponding solvability condition for the right-hand side would be that $\mathbf{u}_{N}^{\mathrm{T}}\left(\tilde{\mathbf{b}}+\mathcal{Z} \mathbf{b}^{*}\right)=0$, which can be ensured by choosing

$$
\mathcal{Z}=-\frac{\mathbf{u}_{N}^{\mathrm{T}} \tilde{\mathbf{b}}}{\mathbf{u}_{N}^{\mathrm{T}} \mathbf{b}^{*}},
$$

which makes $c_{N}$ identically zero. The resulting solution for the linear problem would be given by

$$
\mathbf{Q}=\sum_{i=0}^{N-1} c_{i} \mathbf{v}_{i}+\tilde{c}_{N} \mathbf{v}_{N} .
$$

In the above, the sum on the right side does not contain a term for $i=N$ because $c_{N}=0$ by the solvability condition. The second term on the righthand side represents the solution to the homogeneous problem. The constant $\tilde{c}_{N}$ is arbitrary, reflecting the fact that the solution is not unique. This homogeneous solution $\mathbf{v}_{N}$ contains many oscillations, typically on the scale of the interval discretization $\Delta s=1 / N$. Adding different amounts of this solution results in discrete solutions $Q\left(s_{j}\right)$ with different amounts of "smoothness". In particular, we have found that there is a single value of $\tilde{c}_{N}$ which makes the solution very smooth. Since we expect a smooth and well behaved $Q(s)$ on physical grounds, it seems natural to choose $\tilde{c}_{N}$ to make $Q\left(s_{j}\right)$ as smooth as possible. We define the smoothness of the discrete solution $Q\left(s_{j}\right)$ as the norm of the derivative $\left\|\mathbf{Q}^{\prime}\right\|$ where

$$
\left\|\mathbf{Q}^{\prime}\right\|=\sqrt{\frac{1}{N} \sum_{k=1}^{N}\left(\frac{Q_{k}-Q_{k-1}}{\Delta s}\right)^{2}},
$$

and choose $\tilde{c}_{N}$ to minimize $\left\|\mathbf{Q}^{\prime}\right\|$.

While the above method is appropriate if $\sigma_{N}$ is exactly zero, in our case $\sigma_{N}$ is only approximately zero (usually in the range $10^{-5}-10^{-10}$, depending on $N$ ) because we are solving a discrete version of the integral equation. Thus, the solution to the homogeneous problem is not quite exact, and so (64) is only approximate. Technically, for non-zero $\sigma_{N}$, the self-consistent relation between $\mathcal{Z}$ and the amplitude of the last mode $\tilde{c_{N}}$ is

$$
\mathcal{Z}=-\frac{\mathbf{u}_{N}^{\mathrm{T}} \mathbf{b}^{*}}{\mathbf{u}_{N}^{\mathrm{T}} \tilde{\mathbf{b}}}+\sigma_{N} \frac{\tilde{c}_{N}}{\mathbf{u}_{N}^{\mathrm{T}} \tilde{\mathbf{b}}},
$$

so when we choose a specific $\tilde{c_{N}}$ to obtain a smooth solution, $\mathcal{Z}$ is actually perturbed slightly from the value given by the strict solvability condition (64) by an amount proportional to $\sigma_{N}$. The effect on $\mathcal{Z}$ is negligible for all practical purposes. The primary effect is that it allows us to satisfy the non-linear system of equations "exactly" for finite $N$ even if $\sigma_{N}$ is not exactly zero. If we were to use the strict solvability condition (64) then the errors in satisfying the non-linear equations would be of the same size as the error associated with the solution to the homogeneous linear problem, of order $\sigma_{N}$. Since $\sigma_{N} \rightarrow 0$ as $N \rightarrow \infty$, there is really no difference between either expression for $\mathcal{Z}$. The important result is that at each iteration we obtain a smooth solution and the associated $\mathcal{Z}$ dictated by the solvability condition.

As the iteration scheme proceeds, $\mathbf{Q}$ and $\mathcal{Z}$ each converge. We stop the iteration scheme when the relative change in $\mathbf{Q}$ is less than a prescribed tolerance,

$$
\frac{\left\|\mathbf{Q}-\mathbf{Q}^{0}\right\|}{\|\mathbf{Q}\|}<\text { tol }
$$

where

$$
\|\mathbf{Q}\|=\sqrt{\frac{1}{N+1} \sum_{k=0}^{N}\left(Q_{k}\right)^{2}} .
$$

Unless stated otherwise, we use tol $=10^{-8}$. Our converged solutions satisfy the non-linear system of equations (51) with a residual $\|\mathbf{F}\|$ of less than $10^{-8}$.

The numerical algorithm we use to solve the discretized integral equation (51) is thus summarized as follows:

1. Choose an initial guess $\mathbf{Q}^{0}$;

2. Determine the coefficients $B_{j k}, \tilde{b_{j}}$ and $b_{j}^{*}$ of the linear problem (58);

3. Find the singular value decomposition of $\mathbf{B}$; 
4. Identify the near-zero singular value $\sigma_{N}$ and corresponding singular vector $\mathbf{v}_{N}$ as a solution to the homogeneous matrix problem;

5. Calculate improved solution $\mathbf{Q}$ from (65) by choosing ${\tilde{c_{N}}}_{\text {to }}$ minimize $\left\|\mathbf{Q}^{\prime}\right\|$ with self-consistent $\mathcal{Z}$ given by (67); and

6. Check for convergence using (68). If the solution has not converged let $\mathbf{Q}=\mathbf{Q}^{0}$ and repeat steps 2-6. If solution has converged and solves the equations, accept it as the solution to the nonlinear system.

\section{RESULTS}

Figure 3 shows a typical dendrite shape as calculated with our theory. Each of the locally valid solutions is shown along with the composite solution for the entire shape. For purposes of illustration, we have used the axisymmetric tail solution $R_{\text {tail }}$ from (15). The composite shape for the dendrite is described well by the inner solution for up to about 10 diffusion lengths behind the tip. The composite solution deviates noticeably from a parabolic tip within 1 diffusion length of the tip. The composite and tail solutions approach each other well below the range of $\zeta$ shown because the smallness of $\epsilon$ and $k$ appearing in the tail solution (13).

To demonstrate the relevance of our asymptotic/ numerical solutions to the growth of dendritic arrays during directional solidification, we present a comparison of the predictions of our theory to experimental observations on dendritic growth morphologies by Somboonsuk, Mason and Trivedi (SMT) [21]. In these experiments, an alloy of SCNacetone was directionally solidified between two closely spaced plates. The tip radius $\rho$ and dendrite spacing $\lambda_{x}$ were measured as a function of the temperature gradient and solidification velocity. From the experimental data, we can directly evaluate the parameters $\delta$ and $\Lambda_{x}$. We can also evaluate $\Lambda_{y}$ by assuming that the plate separation corresponds to $\lambda_{y}$. These three parameters determine the solution in our theory, which has a unique tip radius and tip undercooling. Here we present comparisons of tip radius measurements. $\dagger$

Table 1 summarizes the solidification conditions and dendrite measurements from the SMT experiments. Table 2 gives the corresponding non-dimensional input parameters which appear in our theory, along with the measured value of the scaled tip radius $P_{\exp }$ and our calculated values for the tip radius $P_{\text {theory }}$ and the tip position $\mathcal{Z}_{\text {theory. }}$.

Figures 4 and 5 summarize the comparison of our theory with the measured data. Figure 4 com-

†Experimental data on the tip undercooling was not reported so no similar comparison can be made for the tip undercooling.

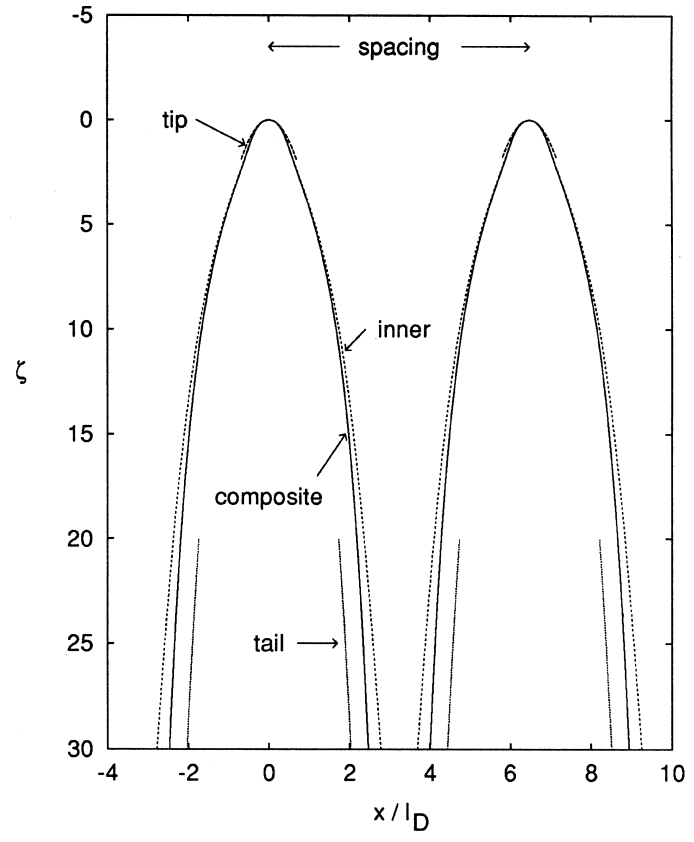

Fig. 3. Calculated dendrite shape. The composite solution consists of appropriately matched tip, inner, and tail solutions. The horizontal scale is expanded to show the details of the slender shape. The tail solution is taken to be axisymmetric for purposes of illustration (see text). Input parameter values are $k=0.1, \epsilon=0.0116, \Lambda_{x}=6.47$, $\Lambda_{y}=7.75$. This solution has a scaled tip radius $P=10.8$ and a scaled tip position $\mathcal{Z}=2.24$.

pares the data at fixed nominal temperature gradient $G=67 \mathrm{~K} / \mathrm{cm}$ with varying velocity (data A0 E0). The agreement of theory and experiment is quite good at small $\epsilon$ (where the asymptotic theory for $\epsilon \ll 1$ is expected to be valid). At larger values of $\epsilon$ the theory gives a tip radius which is smaller than the experimental value by a factor of $2-3$. Figure 5 shows the comparison of theory and experiment at fixed nominal velocities $V=65 \mu \mathrm{m} / \mathrm{s}$ (data B1-B5) and $V=10 \mu \mathrm{m} / \mathrm{s}$ (data D1-D5) with varying temperature gradient. As with the comparison at fixed temperature gradient in Fig. 4, the theory and experiment agree well for small $\epsilon$ but the predicted tip radius is too small by a factor of $1.5-2$ for larger values of $\epsilon(>0.03)$.

We emphasize that our theory contains no adjustable parameters. That our theory gives a tip radius near the experimental measurements of dendrites without regard to surface energy considerations is a remarkable result. The implication of our theory is that, for given dendrite spacings and experimental conditions, there is a selected tip radius and tip undercooling for the dendrite which comes about due to the non-linear interactions in the array. This selection of the tip radius is independent of the surface energy, and thus the selection mechanism is fundamentally different from the marginal stability [4], microsolvability [5,6], and interfacialwave [7] theories for tip selection in single-com- 
Table 1. Data from SCN-acetone experiments. Results are taken from Table 2 of SMT for the velocity range $4 \mu \mathrm{m} / \mathrm{s} \leqslant V \leqslant 100 \mu \mathrm{m} / \mathrm{s}$, above the cell-to-dendrite transition at $V \approx 1 \mu \mathrm{m} / \mathrm{s}$. If two measurements were reported for a given experimental condition we have listed the average measurement. The published data is for plate separations of either 150 or $500 \mu \mathrm{m}$, but the plate spacing for each measurement is not listed. Figure 5 of SMT suggests that most of the measurements are for $150 \mu \mathrm{m}$, so we set $\lambda_{y}=150 \mu \mathrm{m}$. Each data point is labeled

\begin{tabular}{lccccc}
\hline Label & $V(\mu \mathrm{m} / \mathrm{s})$ & $G(\mathrm{~K} / \mathrm{cm})$ & $\lambda_{x}(\mu \mathrm{m})$ & $\lambda_{y}(\mu \mathrm{m})$ & $\rho(\mu \mathrm{m})$ \\
\hline A0 & 100 & 67.0 & 96.8 & 150.0 & 2.0 \\
B0 & 65.6 & 65.6 & 125.2 & 150.0 & 2.3 \\
C0 & 25.5 & 66.7 & 174.2 & 150.0 & 4.0 \\
D0 & 10.7 & 66.85 & 240.25 & 150.0 & 6.25 \\
E0 & 3.97 & 68.0 & 326.0 & 150.0 & 10.05 \\
B1 & 65.3 & 29.6 & 181.0 & 150.0 & 2.86 \\
B2 & 65.8 & 42.4 & 150.0 & 150.0 & 2.56 \\
B3 & 65.9 & 52.0 & 143.0 & 150.0 & 2.30 \\
B4 & 65.0 & 65.6 & 129.8 & 150.0 & 2.455 \\
B5 & 64.3 & 77.0 & 370.0 & 150.0 & 2.0 \\
D1 & 10.6 & 29.9 & 332.5 & 150.0 & 5.25 \\
D2 & 10.6 & 265.0 & 150.0 & 5.0 \\
D3 & 10.6 & 245.25 & 150.0 & 5.73 \\
D4 & 10.5 & 205.95 & 150.0 & 5.27 \\
D5 & 10.8 & 63.7 & 150.0 & 5.40 \\
\hline
\end{tabular}

ponent dendrites. While we do not claim that our model is universally appropriate, the results so far suggest that it may be useful for describing mainstream dendritic growth in a band of velocities that are (1) sufficiently above the cell-to-dendrite transition at low velocities and (2) sufficiently below the high velocity regime where the capillary length and the tip radius become comparable and for which the effect of surface energy on the tip undercooling is not negligible.

Figures 6 and 7 summarize the tip characteristics of solutions to our model as a function of the control parameter $\epsilon$ and the dendrite spacing. For the figures, we assume that $\lambda=\lambda_{x}=\lambda_{y}$. For a given experiment we would know $\epsilon$. Each $\epsilon=$ constant curve corresponds to a family of solutions parameterized by the spacing. For each spacing our theory predicts a unique tip radius and undercooling.

Figure 6 shows the variation of the non-dimensional tip radius (Peclet number) as a function of the spacing for different values of $\epsilon$. For a given value of $\epsilon$, the tip radius generally increases with the dendrite spacing. For nonzero $\epsilon$, each curve on Fig. 6 terminates at a critical value of $\Lambda$ because of a breakdown in the slender-body scalings we have used. In particular, following the $\epsilon=0.01$ family of solutions upwards for increasing $\Lambda$, the corresponding dendrite shape develops a bulb-like tip which then pinches off where the family of solutions terminates. As $\epsilon$ decreases this breakdown of the scalings is pushed to larger values of $\Lambda$, but it does not disappear. Thus, in the context of our asymptotic theory, it is not possible to find solutions for finite $\epsilon$ as $\Lambda \rightarrow \infty$ because our scalings break down. However, it does appear that for small $\epsilon$ the tip radius tends to a constant as $\Lambda$ becomes large before the scalings fail. Figure 6 also shows that for a fixed spacing $\Lambda$, the tip radius approaches a limiting value as $\epsilon \rightarrow 0$. Finally, we note that for small spacings the tip radius is insensitive to $\epsilon$ and varies roughly like $\rho \approx 0.01 \lambda$. It is not feasible to investigate the limit of zero spacing in our model because the infinite sums in (21) converge too slowly.

Figure 7 shows the variation of the non-dimensional tip undercooling as a function of the spacing for different values of $\epsilon$. For small $\Lambda$ the undercooling increases with spacing. Each curve has a maximum undercooling followed by the termination of

Table 2. Non-dimensional parameters and results. The non-dimensional parameters are determined from the experimental data of Table 1 using the materials parameters listed in SMT: $D=1.27 \times 10^{-5} \mathrm{~cm}^{2} / \mathrm{s}, C_{\infty}=5.5 \mathrm{~mol}$ pct acetone, $m_{\mathrm{L}}=-2.22 \mathrm{~K} / \mathrm{mol}$ pct acetone, $k=0.10, T_{\mathrm{m}}=331.24 \mathrm{~K}$ and with $T_{0}=T_{\mathrm{m}}+m_{\mathrm{L}} C_{\infty}$. The theoretical results are determined from our numerical solution to the integral equation with $N=160$ points. The same results are obtained with $N=80$ points to within one unit of the precision indicated

\begin{tabular}{|c|c|c|c|c|c|c|c|}
\hline Label & $\epsilon$ & $\delta$ & $\Lambda_{x}$ & $\Lambda_{y}$ & $P_{\exp }$ & $P_{\text {theory }}$ & $\mathcal{Z}_{\text {theory }}$ \\
\hline A0 & 0.00774 & 0.206 & 7.62 & 11.8 & 20.3 & 17.3 & 3.31 \\
\hline B0 & 0.0116 & 0.224 & 6.47 & 7.75 & 10.3 & 10.8 & 2.24 \\
\hline $\mathrm{C} 0$ & 0.0302 & 0.286 & 3.50 & 3.01 & 2.66 & 2.2 & 0.92 \\
\hline D0 & 0.0722 & 0.380 & 2.02 & 1.26 & 0.729 & 0.43 & 0.61 \\
\hline E0 & 0.198 & 0.617 & 1.02 & 0.469 & 0.159 & 0.06 & 0.69 \\
\hline B1 & 0.00524 & 0.190 & 9.31 & 7.71 & 28.1 & 19.8 & 4.42 \\
\hline B2 & 0.00745 & 0.204 & 7.77 & 7.77 & 17.8 & 14.7 & 3.26 \\
\hline B3 & 0.00912 & 0.213 & 7.42 & 7.78 & 13.1 & 13.0 & 2.76 \\
\hline B4 & 0.0117 & 0.225 & 6.64 & 7.68 & 10.8 & 11.0 & 2.21 \\
\hline B5 & 0.0138 & 0.234 & 6.07 & 7.59 & 8.78 & 9.6 & 1.90 \\
\hline D1 & 0.0326 & 0.292 & 3.09 & 1.25 & 1.34 & 0.6 & 0.6 \\
\hline D2 & 0.0447 & 0.322 & 2.78 & 1.25 & 1.07 & 0.53 & 0.64 \\
\hline D3 & 0.0509 & 0.336 & 2.21 & 1.25 & 0.864 & 0.48 & 0.64 \\
\hline D4 & 0.0701 & 0.376 & 2.03 & 1.24 & 0.637 & 0.43 & 0.61 \\
\hline D5 & 0.0824 & 0.401 & 1.75 & 1.28 & 0.591 & 0.40 & 0.60 \\
\hline
\end{tabular}




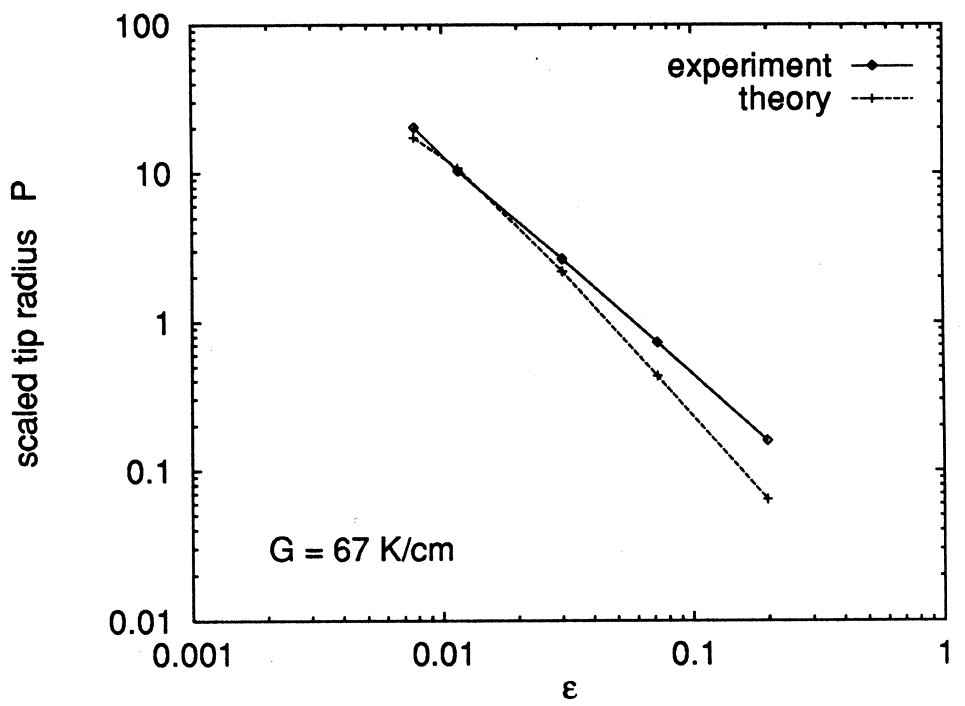

Fig. 4. Comparison of theoretical predictions for the scaled tip radius $P$ to the experimental data. Results are for nominal temperature gradient $G=67 \mathrm{~K} / \mathrm{cm}$ (data A0-E0). The theory assumes that $\epsilon$ is small.

the family of solutions due to the breakdown of our scalings as mentioned above. Corresponding to the behavior in Fig. 6, the point of termination moves to larger $\Lambda$ as $\epsilon$ decreases. Further, the maximum of the curve also moves to larger $\Lambda$ and becomes less sharp as $\epsilon$ decreases. We do not know if the feature of a maximum in the undercooling curves corresponds to a physical effect, or, because it occurs relatively close to where a family of solutions termi- nates, it is an indication that the scalings are starting to fail.

The information from Figs 6 and 7 is combined in Fig. 8 to show how the non-dimensional undercooling is related to the Peclet number of the tip for different values of $\epsilon$. For each curve, the spacing is increasing as the Peclet number increases. In general, the trend is that the tip undercooling increases with Peclet number, then passes through a maximum at Peclet number $\approx 0.15$ before the solution

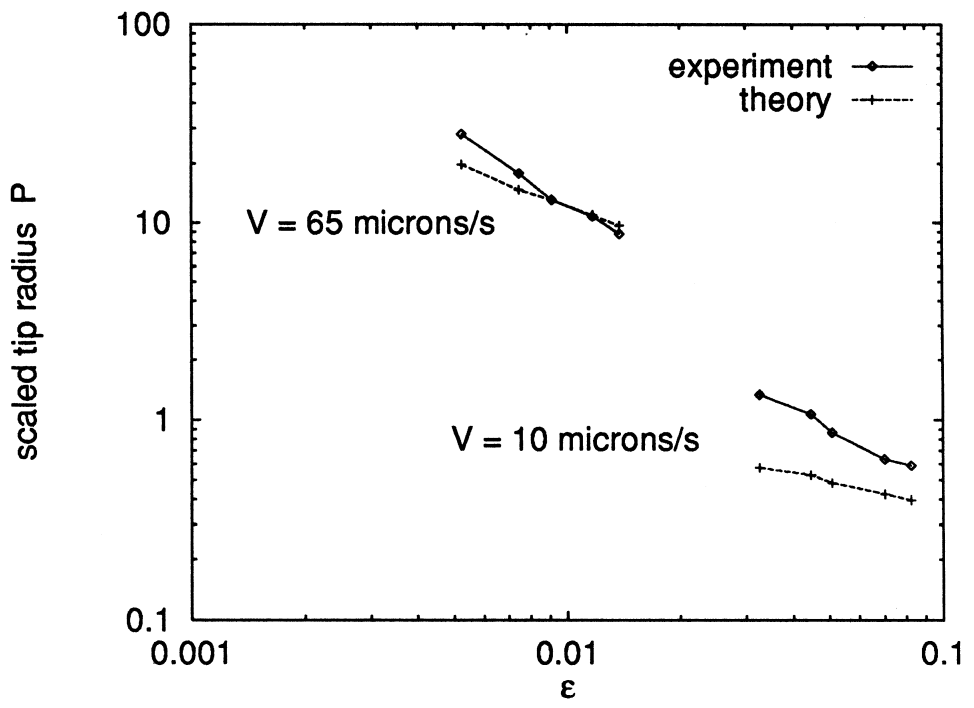

Fig. 5. Comparison of theoretical predictions for the scaled tip radius $P$ to the experimental data. Results are for nominal velocity $V=65 \mu \mathrm{m} / \mathrm{s}$ (data B1-B5) and nominal velocity $V=10 \mu \mathrm{m} / \mathrm{s}$ (data D1-D5). The theory assumes that $\epsilon$ is small. 


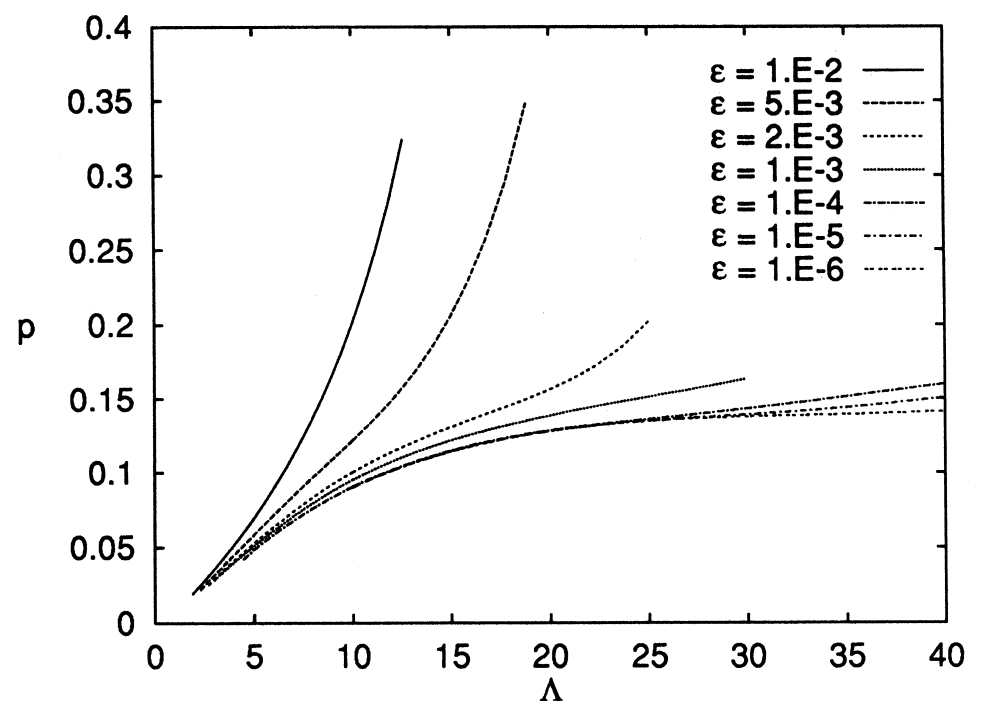

Fig. 6. Theoretical calculations of the non-dimensional tip radius $p=\rho / l_{\mathrm{D}}$ as a function of the dendrite spacing $\Lambda=\lambda / l_{\mathrm{D}}$ for different values of the control parameter $\epsilon$.

family terminates at larger Peclet number. Since our theory is based on $p \ll 1$, it may very well be true that the maxima of the undercooling curves (which is the same feature present in Fig. 7), and the subsequent termination of the solution family is because the Peclet number of the solutions becomes too large to be described by our theory.

For reference, we have also plotted on Fig. 8 the undercooling relationship for the Ivantsov dendrite [8], $\Theta=(p / 2) E_{1}(p / 2) \exp (p / 2)$. In our theory we assume $p=O(\epsilon)$ and neglect corrections of $O(\epsilon)$. Thus, in the context of our small Peclet number theory, the Ivantsov relation would be given by $\Theta \backsim(p / 2)\left(-\ln (p / 2)-\gamma_{\mathrm{E}}\right)$. We note that as $\epsilon \rightarrow 0$ there is a collapse of our solution curves onto the small Peclet number Ivantsov relation. Thus, in this sense, we recover the Ivantsov solution in the limit $\epsilon \rightarrow 0$. However, the collapse of the curves onto the Ivantsov solution does not hold for Peclet number $\rightarrow 0$. The reason is because, according to Fig. 6 , as $p \rightarrow 0$, the dendrite spacings are necessarily going to zero as well. Thus, as $p \rightarrow 0$ in Fig. 8, the solutions become less Ivantsov-like because the neighboring dendrites are actually getting closer. So it is not surprising that the Ivantsov relation is not recovered as $p \rightarrow 0$.

Since $\epsilon$ measures the relative strength of the temperature gradient, we can interpret our $\epsilon \rightarrow 0$ results in terms of isothermal dendrite growth. In particular, the periodic boundary conditions for a unit cell of our array are equivalent to having side walls for the cell boundary. Thus, in the limit of $\epsilon \rightarrow 0$ our

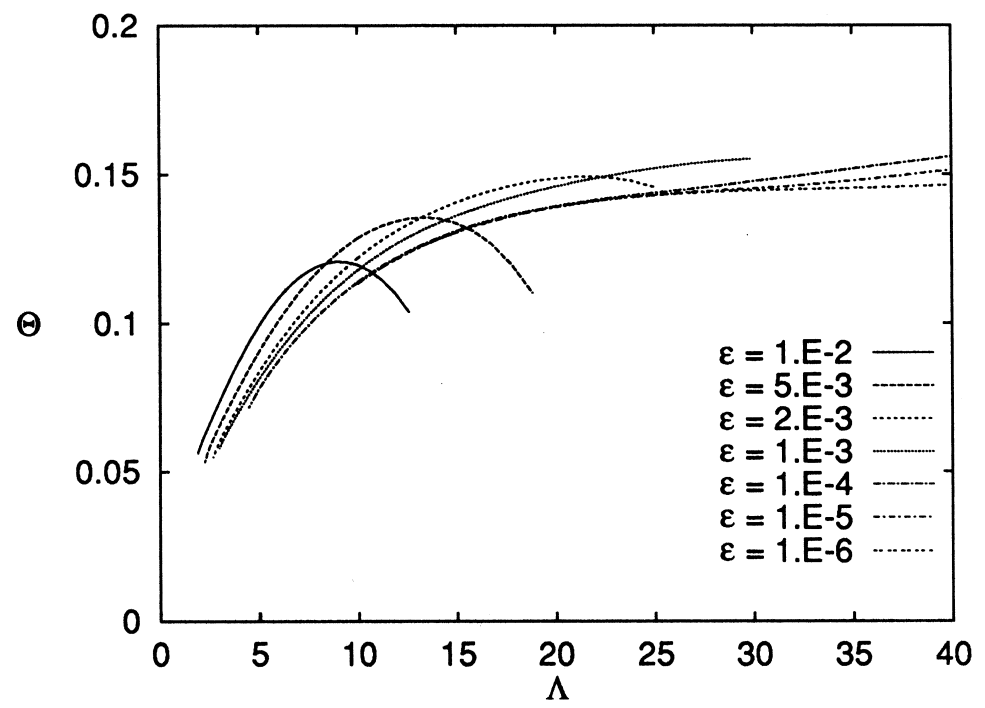

Fig. 7. Theoretical calculations of the non-dimensional tip undercooling $\Theta=\Delta T / k \Delta T_{0}$ as a function of the dendrite spacing $\Lambda=\lambda / l_{\mathrm{D}}$ for different values of the control parameter $\epsilon$. 


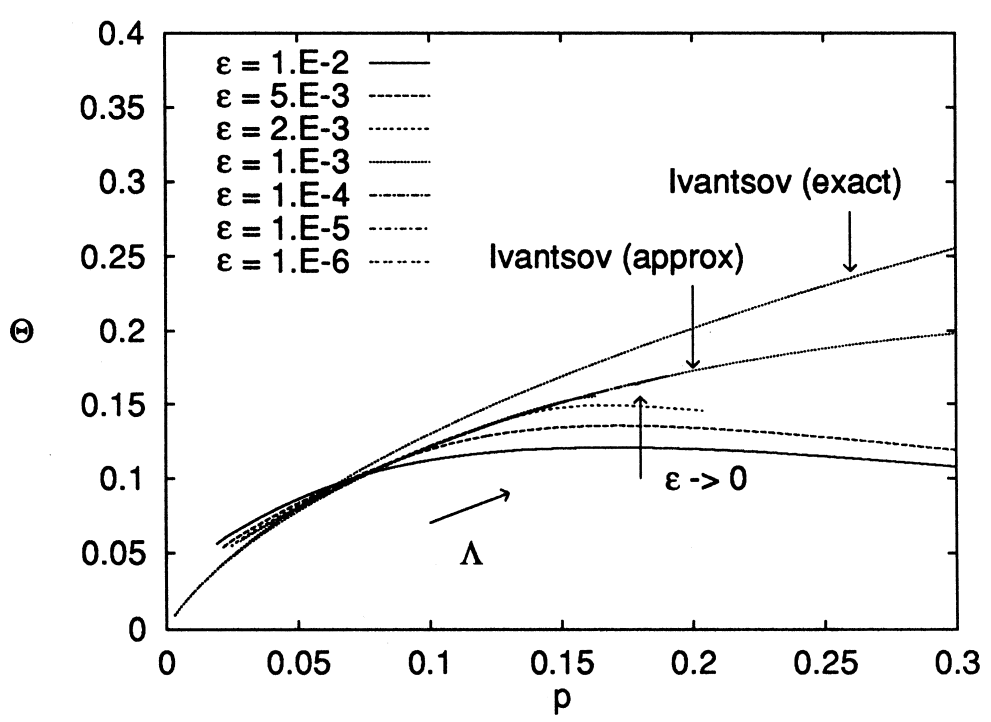

Fig. 8. Theoretical calculations of the non-dimensional tip undercooling $\Theta$ as a function of the nondimensional tip radius $p$ for different values of the control parameter $\epsilon$. Along each curve the spacing $\Lambda$ increases as $p$ increases.

theory describes the growth of a single isothermal solute dendrite along the axis of a tube. In our array geometry, $\lambda$ would be the side length of a square tube. In an experiment, we would impose the undercooling $\Theta$ and measure the tip radius and growth velocity. In our theory, if $\Theta$ is known then $\Lambda=\lambda V / D$ is prescribed from Fig. 7. Since we know $D$ and the dimensions of the tube, $\Lambda$ gives us the growth velocity as $V=\Lambda D / \lambda$. From $\Lambda$ we also find $p=\rho V / D$ from Fig. 6 which determines the tip radius as $\rho=p D / V$. Thus, using Figs $6-8$, the pre- dictions of our theory in the case of $\epsilon \rightarrow 0$ could be tested directly by experiments in which isothermal solute dendrites of known undercooling are grown in square tubes.

In our comparison to the experimental data we used the experimentally measured spacings to predict the tip radius. In our model, for a given set of solidification conditions there exists a family of steady-state solutions parameterized by the spacings of the array. Our steady-state theory thus provides no direct means of determining which spa-

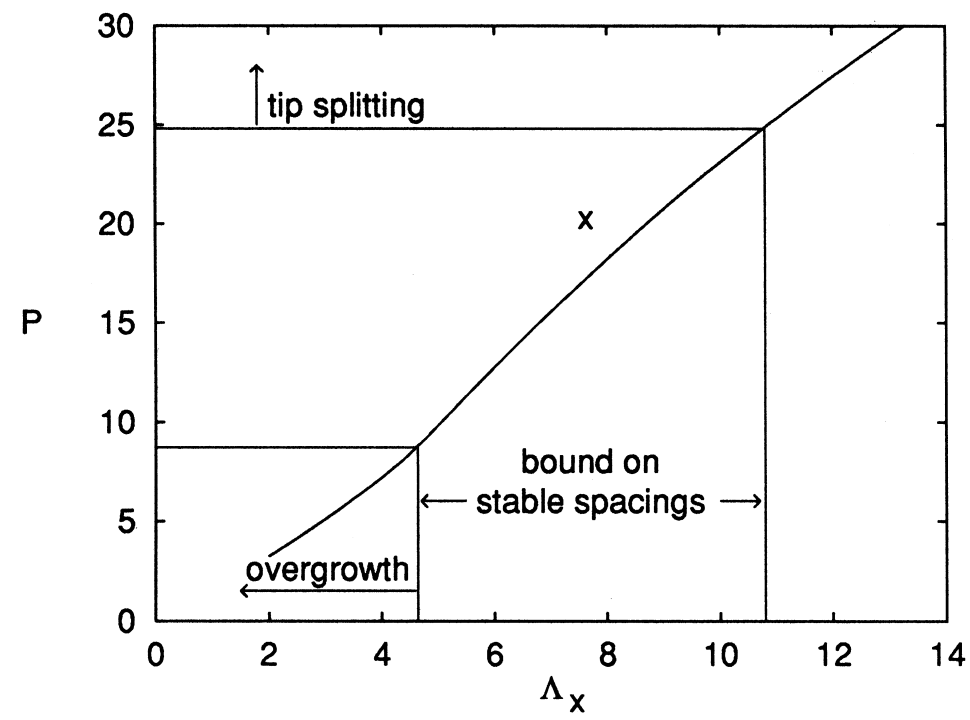

Fig. 9. A bound on the range of stable spacings. The solid curve shows the variation of the tip radius parameter $P$ with the dendrite spacing parameter $\Lambda_{x}$ in our theory for the solidification conditions of data point A0. Also shown is a lower bound for array stability given by the Warren-Langer theory for array overgrowth [9], and an upper bound for the tip radius from the marginal stability theory [4]. The maximum stable tip radius translates to an upper bound for stable array spacings. The single exper-

imental data point $(\mathrm{x})$ is indicated within the stability bounds and close to our theoretical curve. 
cing will be observed in practice. This is not surprising, since the selection of dendrite spacings is an intrinsically time- and history-dependent phenomenon $[22,13,14]$. However, our steady-state theory contains some features which may be relevant to determining the range of stable dendrite spacings which exist for a given set of experimental conditions $[13,14]$. To illustrate this idea, we consider how the predictions of our theory depend on the dendrite spacing. The variation of the tip radius parameter $P$ with the dendrite spacing parameter $\Lambda_{x}$ for data point $\mathrm{A} 0$ is shown in Fig. 9. As with the general trend displayed in Fig. 6, the tip radius increases with increasing dendrite spacing.

To determine which array spacings are stable we consider the instability mechanisms which would affect the dendrite array [13] in the context of our model. First, if the dendrites are too close together, then the competition between the tips can cause some members of the array to be overgrown. This gives a lower bound on stable array spacings. Second, if the tip radius becomes too large, then the dendrite tip is unstable to tip splitting. Since our results have the critical feature that the tip radius increases with dendrite spacing, a maximum stable tip radius gives an upper bound on array spacings which can be stable. While the rigorous analysis needed to predict these upper and lower stability bounds in the context of our model has not yet been carried out, we can employ the results of related models to illustrate how the above ideas give a range of stable array spacings which are relevant to experiments.

For the lower stability bound, we note that our approach is similar to the description of steadystate arrays in Warren and Langer [9]. The primary difference is that they assume that the dendrite shape is parabolic, whereas we solve for the shape. They also assume that the tip radius is determined by a selection condition based on the surface energy and involving the parameter $\sigma^{*}$, whereas our tip radius is determined by the array spacing. Aside from these fundamental philosophical differences, the results are similar in that both give a family of steady-state solutions parameterized by the array spacing. In their paper, Warren and Langer examined the stability of an array with respect to the overgrowth mechanism, and found that there was a critical spacing above which the array was stable. Although we have not yet evaluated the stability of our array solutions using the approach set forth in Warren and Langer, we do expect that a similar stability bound will be present. For purposes of illustration, we have indicated where the WarrenLanger stability boundary would be in Fig. 9 $\left(\Lambda_{x}=4.6\right)$, based on the results presented in Fig. 4 of Warren and Langer. Array spacings smaller than this spacing are unstable to the overgrowth instability, while array spacings greater than this spacing are stable with respect to the overgrowth of neighboring dendrites.

To determine an upper stability bound for array spacings, we consider the marginal stability condition for a dendrite tip [4]. While the marginal stability condition of [4] applies to a single-component dendrite, this condition can be readily translated to an equivalent condition for directional solidification of a binary alloy (see equation (2.18) of [9]). In terms of our scalings, the marginal stability condition gives a maximum stable tip radius approximated by

$$
P^{*}=\frac{1}{\epsilon} \sqrt{\frac{2 l_{\mathrm{c}}}{l_{D} \sigma^{*}}},
$$

where $\sigma^{*}=0.025$, and we have dropped corrections of order $\epsilon$. For the experimental conditions of data point $\mathrm{A} 0$, the maximum stable tip radius is $P^{*}=24.8$. For purposes of comparison, we have indicated this maximum stable tip radius on Fig. 9. Based on our relationship between tip radius and array spacing, the maximum stable tip radius corresponds to an upper stability bound for the array of about $\Lambda_{x}=10.7$. Stable tip radii and spacings are expected to lie on the theoretical curve between the two stability boundaries shown, and it is seen that the data point lies within the range of stability and close to the theoretical curve.

Thus, using the two stability bounds from other theories in conjunction with our relationship between the tip radius and the dendrite spacing puts a bound on the stable array spacings. Over this range of stable spacings there is a surprisingly large variation in the predicted tip radius. While the precise determination of the stability bounds for Fig. 9 using the scalings and equations of our model may change the stability bounds somewhat from the Warren-Langer and marginal stability bounds, we expect that the essential feature of an upper and lower bound on the range of stable spacings will remain.

In the Introduction we mentioned that the selection of a tip radius and tip undercooling by the spacing was due to the presence of interaction effects between dendrites. Such selection was not found in the limiting case for which the spacing was much less than the diffusion length as studied for cellular morphologies $[15,16]$. The cellular work and our results appear to be contradictory because, in the absence of surface energy considerations the cellular solutions have two degrees of freedom but our solutions have only one degree of freedom. Unfortunately, a direct comparison between our dendrite theory and these cellular theories is not possible because each involves different specific assumptions about the relative sizes of length scales to formulate asymptotic solutions. We employ slender body theory to describe dendrites for which the diffusion length $l_{\mathrm{D}}$ is much smaller than the thermal 
length $l_{\mathrm{T}}$ and for which the non-dimensional undercooling is small. The cellular work is concerned with "fat" finger solutions which occur for $l_{\mathrm{D}} /$ $l_{\mathrm{T}}=O(1)$ and for which the non-dimensional undercooling is order unity. Despite this discrepancy in length scale assumptions, however, it is possible to show how the presence of selection in our results does not contradict the absence of selection in the cellular results. In the limit of small spacings, the solutions to our integral equation recover the two degrees of freedom exhibited by the cellular solutions.

We reconsider our integral equation (19) in the limit of small spacings. We rescale the parameters and unknowns using $l_{\mathrm{D}} / l_{\mathrm{T}}=\epsilon / \delta \ll 1, \quad \lambda / l_{\mathrm{D}}=\delta^{1 / 2} \tilde{\Lambda}$, $q(\zeta)=\delta \tilde{q}(\zeta)$, with other variables scaled as before and $\delta=1 / \ln (1 / \epsilon) \ll 1$. To leading order in $\delta$, the second term on the right hand side of (19) and the integral involving $G_{\text {near }}$ do not appear. Further, the integral involving $G_{\text {array }}$ in (19) can be approximated for small spacings using the asymptotic results in Appendix $\mathrm{C}$ of [23]. To leading order, the integral equation becomes

$$
\begin{aligned}
\mathcal{Z}+\zeta= & \frac{\tilde{q}(\zeta)}{4 \pi}+\frac{1}{\tilde{\Lambda}_{x} \tilde{\Lambda}_{y}}\left(\int_{0}^{\zeta} \tilde{q}\left(\zeta^{\prime}\right) \mathrm{d} \zeta^{\prime}\right. \\
& \left.+\int_{\zeta}^{\infty} \tilde{q}\left(\zeta^{\prime}\right) \exp \left(\zeta-\zeta^{\prime}\right) \mathrm{d} \zeta^{\prime}\right)
\end{aligned}
$$

The exact solution to the integral equation is

$$
\tilde{q}(\zeta)=\tilde{\Lambda}_{x} \tilde{\Lambda}_{y}\left\{1+\mu\left[\mathcal{Z}-1-\frac{\tilde{\Lambda}_{x} \tilde{\Lambda}_{y}}{4 \pi}\right] \exp (-\mu \zeta)\right\}
$$

where

$$
\mu=-1 / 2+\sqrt{1 / 4+4 \pi / \tilde{\Lambda}_{x} \tilde{\Lambda}_{y}} .
$$

In contrast to the numerical solutions for the line source $q(\zeta)$ in which the tip undercooling $\mathcal{Z}$ is determined by a solvability condition, here the line source strength $\tilde{q}(\zeta)$ has an extra degree of freedom associated with the unspecified tip undercooling $\mathcal{Z}$. Thus, we find that if we consider our integral equation in the limit of small spacings then there is an additional degree of freedom in the solutions which corresponds to the findings on cellular solutions in the limit $\lambda / l_{\mathrm{D}} \ll 1, l_{\mathrm{D}} / l_{\mathrm{T}}=O(1)$ and $z_{\text {tip }} /$ $l_{\mathrm{D}}=O(1)[15,16]$. The selection mechanism in our model is due to the dendrite spacings being finite and disappears in the limit of small spacings.

Another point mentioned in the Introduction is that our model is based on the idea of using smooth needle crystal dendrites to describe the characteristics of real dendrites. Since real dendrites develop sidebranches, the success of our model in predicting dendrite behavior relies on the assumption that a dendrite with sidebranches has the same "average" behavior as a smooth needle crystal. The encouraging agreement between experiment and theory suggests that at least in some parameter ranges this is a good approximation. This is not to say, however, that sidebranches are irrelevant to dendritic growth. Our results do not necessarily rule out dynamic tip selection mechanisms associated with sidebranch formation.

Our results do not necessarily displace any of the surface-energy-based selection criteria either. To be fair, without surface energy our needle crystals, as with the Ivantsov dendrite, are unstable to small disturbances; for the theory to be truly complete the effect of surface energy should be included. And it is not obvious what surface energy will do to our selection theory. For example, when surface energy is added to the Ivantsov dendrite, smooth solutions only exist if the surface tension is anisotropic. It might be the case that by adding surface tension to our model it will no longer be possible to describe smooth solutions, but this would need to be demonstrated. It is also possible that surface tension may act as it does in the Ivantsov problem and select a tip radius. In this case a selected tip radius would result in the selection of a unique dendrite spacing in our theory.

An important feature associated with real dendrites that we can not reproduce with smooth needle crystals is the true mechanism for determining the upper stability boundary for dendrite spacings. The actual mechanism of instability at large spacings is not tip splitting but the outgrowth of tertiary sidebranches [13], which we can not describe. However, an upper stability boundary determined by tip splitting is still relevant in the sense that it constrains the range of stable spacings from above. Sidebranches or no, it should not be possible to have a tip radius which exceeds the critical radius for tip splitting, so the tip splitting criteria does give useful information about what spacings are definitely not stable. Since the experiments show that the tertiary sidebranch mechanism controls the upper limit on spacings, it is expected that the corresponding stability boundary will be at a smaller spacing than the stability boundary for tip splitting, and will further reduce the range of stable spacings. But since the tertiary mechanism is clearly not a feature of our branchless solutions we make use of the tip splitting mechanism to at least obtain an upper bound on where the upper stability boundary lies.

As a final point, the fact that the integral equation we are solving is non-linear means that there exists the possibility for more than one solution to exist for a given set of parameters. We have found that, in addition to the solution which we have identified as a "dendrite," there is also a distinctly different "type-2" solution for the same set of parameters. This second solution has a much larger tip radius and larger tip undercooling than the "dendrite" solution. Figure 10 shows the solute line source strengths which are determined from the 


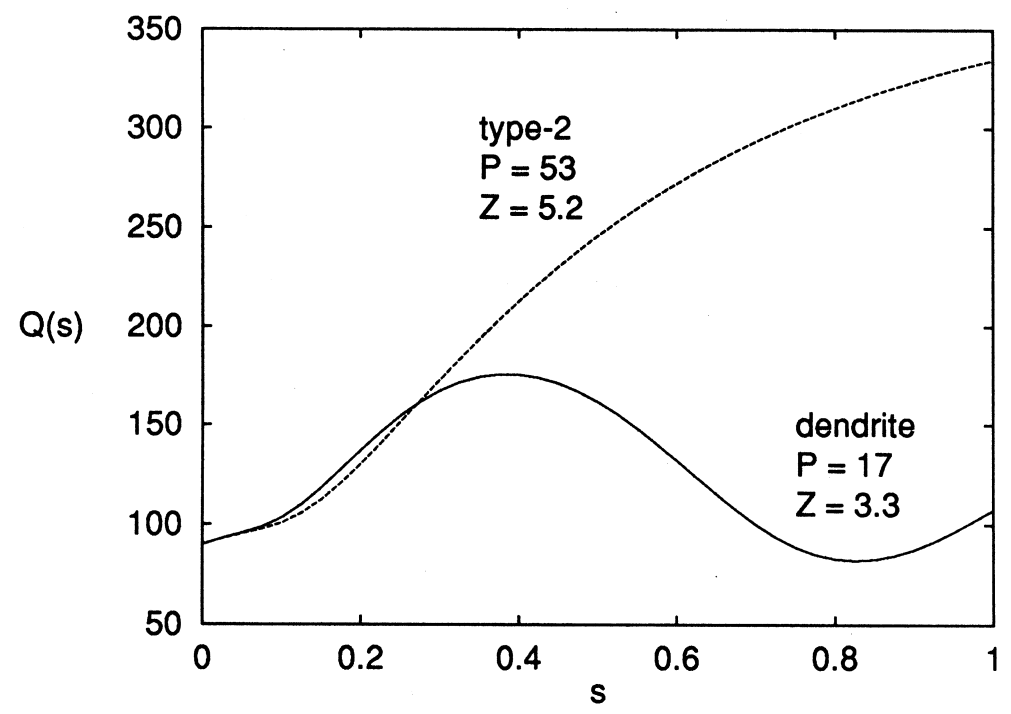

Fig. 10. Two solutions to the integral equation for the experimental conditions of data point B0. Solutions are shown for the solute line source strength as a function of the mapped distance behind the tip. The "dendrite" solution has a tip radius which corresponds closely to the experimental observation; the "type-2" solution has a much larger tip radius and larger tip undercooling.

integral equation (37) for the dendrite and type-2 solutions. Figure 11 shows the difference in the solution shapes. It is tempting to speculate that the type- 2 solution is a vestige of the cellular solidification morphology that has survived well into the dendritic parameter regime. However, even if the type-2 solution is related to the cellular morphology, our model is only appropriate for describing "slender" morphologies away from the cell-todendrite transition which occurs at $\epsilon \approx 1$, so we do not expect our theory to be valid where the transition to cells would occur.

\section{CONCLUSIONS}

An integral equation for the growth of asymptotically slender steady-state arrays of needle crystals during directional solidification has been developed. In this paper we have solved the integral equation numerically to determine the composite solution for

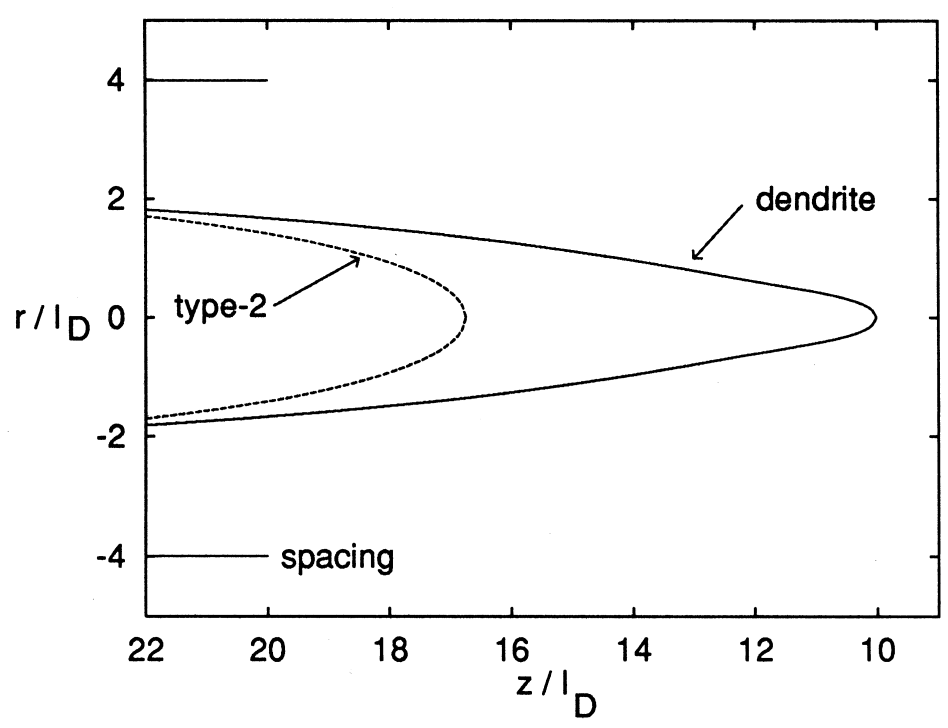

Fig. 11. Composite shapes of the dendrite and type-2 solutions depicted in Fig. 9. Plot aspect ratio is 1:1. The $z / l_{\mathrm{D}}$ coordinate indicates the relative position in the imposed temperature gradient with larger undercooling corresponding to larger $z$. 
the shape of the needle crystal. The model and its solutions were demonstrated to be relevant to dendritic growth at moderate velocities. The solutions have the following features:

- For given solidification conditions and array spacings the non-linear interactions of the dendrites in the array determines a unique tip radius, tip undercooling and dendrite shape. This selection mechanism is fundamentally different from criteria based on surface energy.

- The predictions of our theory are in good agreement with experimental measurements of the dendrite tip radius in the $\mathrm{SCN}$-acetone system for velocities sufficiently above the cell-to-dendrite transition.

- The selected tip radius generally increases with the array spacing, tending to a constant for small temperature gradients and large spacings.

- For finite array spacing, in the limit of a weak temperature gradient, our theory results in the selection of a particular member of the Ivantsov family of solutions.

- Given the solute undercooling, our model can be used to predict a unique tip radius and growth velocity for a single, isothermal solute dendrite grown along the center axis of a square tube.

- The variation of tip radius with array spacing is consistent with a band of stable array spacings. The lower stability bound for array spacings is determined by the criteria for overgrowth of neighboring dendrites. An upper stability bound for array spacings can be determined from the condition for tip splitting.

- In addition to the "dendritic" solution there is also a "type-2" solution with larger tip radius and larger tip undercooling for the same experimental conditions and array spacings. This second solution may be related to the low-velocity cellular morphology.

- Our integral equation has an apparent transition in the number of degrees of freedom in the limit of small spacings. We have not yet been able to describe the precise location of this transition mathematically, nor explain the physical mechanism. An explanation of how this transition in the number of degrees of freedom occurs and a verifi- cation of our description of this new selection mechanism awaits future research.

Acknowledgements - This research is funded by a grant from the EPSRC. We are grateful to Dan Anderson, Roger Bonnecaze, Steve Davis, John Hinch, John Lister, Adam Shultz, Torquil Smith and Grae Worster for many valuable discussions over the course of this work.

\section{REFERENCES}

1. Kurz, W. and Trivedi, R., Acta metall. mater., 1990, 38, 1.

2. Trivedi, R. and Kurz, W., Acta metall. mater., 1994, 42, 15.

3. Spencer, B. J. and Huppert, H. E., Acta metall. mater., 1997, 45, 1535.

4. Langer, J. S. and Müller-Krumbhaar, H., Acta metall., 1978, 26, 1681.

5. Ben Amar M. and Brener, E., Phys. Rev. Lett., 1993, 71, 589.

6. Brener, E., Phys. Rev. Lett., 1993, 71, 3653.

7. Xu, J. J., Phys. Rev. A, 1991, 43, 930.

8. Ivantsov, G. P., Dokl. Akad. Nauk. SSSR, 1947, 58, 567.

9. Warren, J. A. and Langer, J. S., Phys. Rev. A, 1990, 42, 3518

10. Hunt, J. D., in Solidification and Casting of Metals. The Metals Society, London, 1979, p. 3.

11. Kurz, W. and Fisher, D. J., Acta metall., 1981, 29, 11.

12. Billia, B. and Trivedi, R., in Handbook of Crystal Growth, Vol 1, ed. D. T. J. Hurle. Elsevier, Amsterdam, 1993, pp. 899-1074.

13. Han, S. H. and Trivedi, R., Acta metall. mater., 1994, 42, 25.

14. Weidong, H., Xingguo, G. and Yaohe, Z., J. Crystal Growth, 1993, 134, 105.

15. Dombre, T. and Hakim, V., Phys. Rev. A, 1987, 36, 2811.

16. Ben Amar M. and Moussallam, B., Phys. Rev. Lett., 1988, 60, 317.

17. Lu, S-Z. and Hunt, J. D., J. Crystal Growth, 1992, 123, 17.

18. Langer, J. S., Rev. Mod. Phys., 1980, 52, 1.

19. Hinch, E. J., Perturbation Methods, Cambridge University Press, Cambridge, 1992.

20. Hansen, P. C., Inverse Problems, 1992, 8, 849.

21. Somboonsuk, K., Mason, J. T. and Trivedi, R., Metall. Trans. A, 1984, 15A, 967.

22. Warren, J. A. and Langer, J. S., Phys. Rev. E, 1993, 47, 2702.

23. Spencer, B. J. and Huppert, H. E., J. Crystal Growth, 1995, 148, 305. 